\title{
Chromatographic separation of glycated nucleotides
}

\author{
Ivan Mikšík \\ Institute of Physiology, Czechoslovak Academy of Sciences, Videňská 1083, 14220 Prague 4 (Czechoslovakia) \\ Zdeněk Hodný \\ Research Institute of Gerontology, 90101 Malacky (Czechoslovakia)

\section{Zdeněk Deyl} \\ Institute of Physiology, Czechoslovak Academy of Sciences, Videňská 1083, 14220 Prague 4 (Czechoslovakia)
}

(First received July 20th, 1992; revised manuscript received October 14th, 1992)

\begin{abstract}
A high-performance liquid chromatographic method for the separation of nucleotide glycation reaction product(s) has been developed. The product(s) arising during in vitro glycation of a nucleotide (AMP, GMP or CMP) with a reducing sugar (ribose or glucose) were clearly resolved from the non-glycated constituents of the reaction mixture. The peak(s) of the glycated product(s) increased when an amino acid (lysine, arginine, $\beta$-alanine or $\mathrm{N}_{\varepsilon}$-acetyllysine) was added to the reaction mixture. This increase probably corresponds to the formation of a new product with a different absorption maximum ( 250 versus $260 \mathrm{~nm}$ ). Conversely, formation of this product(s) was inhibited by the presence of the metal-chelating agent diethylenetriamine pentaacetic acid and by aminoguanidine.
\end{abstract}

\section{INTRODUCTION}

DNA lesions (e.g. chromosomal aberrations [1], strand breaks [2], cross-linked DNA bases [3], DNA-protein cross-links [4]) are known to accumulate in ageing mammalian cells. Age-related changes in DNA repair [5], replication [6], and transcription [7] have also been observed.

Bucala et al. [8] obtained evidence that nucleic acids, like proteins, can undergo non-enzymic modification by sugars (glycation). Incubation of single-stranded DNA or nucleotides with glucose or glucose-6-phosphate (Glu-6-P) produces spectral changes analogous to those described for

Correspondence to: I. Mikšík, Institute of Physiology, Czechoslovak Academy of Sciences, Videňská 1083, 14220 Prague 4, Czechoslovakia. non-enzymic browning of proteins. Moreover, incubation of viral DNA with Glu-6-P decreased its transcription efficiency in bacterial cells; similar treatment of double-stranded DNA plasmids produced DNA aberrations, including insertions and deletions probably as a result of an effort to repair glycation-induced DNA lesions by the bacterial cell $[9,10]$. It was also demonstrated that Glu-6-P can react with the primary amino group of lysine (or putrescine) to form an unknown reaction intermediate, which can subsequently react very rapidly with single-stranded and doublestranded DNA equally well [11]. Shires et al. [12] suggested possible involvement of three-carbon dicarbonyls in DNA modification in vivo.

The mutagenicity of Maillard reaction products formed during incubation of glucose with amino acids was previously observed for Sal- 
monella typhimurium [13]. Other mutagens formed through the Maillard reaction were identified [14-16].

It seems reasonable that Maillard glycation reaction may affect DNA function in vivo; however, the mechanism of the reaction and the chemical nature of products remain unclear.

Nucleotides and nucleic acid constituents are at present separated by various kinds of highperformance liquid chromatography (HPLC) $[17,18]$, particularly in the reversed-phase mode, and somctimes by affinity (mctal chclate) chromatography. Recently [19], a silica gel chromatographic method with a mobile phase of ethyl acetate and weakly soluble polar components, such as ethylene glycol, triethylene glycol or formamide, was reported for the separation of the highly polar derivatives of purine and pyrimidine.

\section{EXPERIMENTAL}

\section{Chemicals}

Adenosine-5'-phosphate, cytidine-5'-phosphate and guanosine- $5^{\prime}$-phosphate disodium salts (AMP, CMP, GMP), as well as L-lysine were obtained from Serva (Heidelberg, Germany), DL-glyceraldehyde, $\mathbf{N}_{\varepsilon}$-acetyl-L-lysine, diethylenetriamine pentaacetic acid (DETAPAC) and D-ribose were obtained from Sigma (St. Louis, MO, USA), aminoguanidine bicarbonate (AG) was purchased from Koch-Light (Colnbrook, $\mathrm{UK}$ ), and $\beta$-alanine from Loba (Wien-Fischamend, Austria).

\section{Preparation of products}

Nucleotides were incubated in equimolar mixture $(20 \mathrm{~m} M)$ with sugars (DL-glyceraldehyde, Dribose, and D-glucose, respectively) and amino acids (L-lysine, L-arginine, $\mathrm{N}_{\varepsilon}$-acetyl-L-lysine, and $\beta$-alanine, respectively) in $50 \mathrm{~m} M$ sodium phosphate buffer $\left(\mathrm{pH} \mathrm{7.5)}\right.$ for seven days at $37^{\circ} \mathrm{C}$ as follows: nucleotide, sugar and amino acid separately (blanks); nucleotide plus sugar, nucleotide plus sugar plus amino acid; all control combinations (sugar plus amino acid etc.) were also analysed. The same sets of experiments were performed in the presence of $1 \mathrm{~m} M$ DETAPAC or
$20 \mathrm{~m} M$ aminoguanidine. Toluene $(1 \mu \mathrm{l} / \mathrm{ml})$ was added to the incubation mixture to prevent microbial growth.

\section{High-performance liquid chromatography}

HPLC was carried out with a Waters automated gradient controller (Millipore, Milford, MA, USA) with a Waters Model 510 pump and a Spectra-Physics SP 4290 integrator (San Jose, CA, USA). A steel column $(250 \times 8 \mathrm{~mm}$ I.D. $)$ packed with Separon SGX $7 \mu \mathrm{m}$ (Tessek, Prague, Czcchoslovakia) and a glass precolumn $(30 \times 3$ mm I.D.) packed with Separon SGX $5 \mu \mathrm{m}$ were mounted in the instrument. The sample $(5 \mu 1)$ was injected into the column and eluted under isocratic conditions with methanol-ethylene glycol-ethyl acetate-water $(98: 100: 420: 68, \mathrm{v} / \mathrm{v})$ at a flow-rate of $1.6 \mathrm{ml} / \mathrm{min}$. The eluate was monitored at $254 \mathrm{~nm}$ using a Waters Model 441 absorbance detector. The temperature was $36 \pm 0.5^{\circ} \mathrm{C}$. For spectral measurements of separated peaks, an HP 1090 liquid chromatograph with a diodearray detector (Hewlett-Packard, Amstelveen, Netherlands) was used.

\section{Thin-layer chromatography}

Thin-layer chromatography was carried on HPTLC silica gel $60 \mathrm{~F}_{254}$ plates $(10 \times 10 \mathrm{~cm})$ for nano TLC (Merck, Darmstadt, Germany). The plates were developed with $n$-propanol-methanol-ammonia-water $(3: 1: 1: 1, \mathrm{v} / \mathrm{v})$ in a $11 \times 12$ $\times 4 \mathrm{~cm}$ chamber (saturated for $2 \mathrm{~h}$ ). The compounds were detected as quenching spots under UV light at $254 \mathrm{~nm}$ (Min UVIS; Desaga, Heidelberg, Germany).

\section{RESULTS AND DISCUSSION}

A chromatographic system for the separation of the products arising during Maillard reaction of nucleotides with sugars was developed. The retention times of unreacted nucleotides in this system are very long ( $>80 \mathrm{~min}$ ) and therefore the corresponding peaks are not shown in the figures. The peak with a retention time of $5.5 \mathrm{~min}$ and the negative peak at the retention time $11.0 \mathrm{~min}$ result from the sample buffer. The next peak (in the 


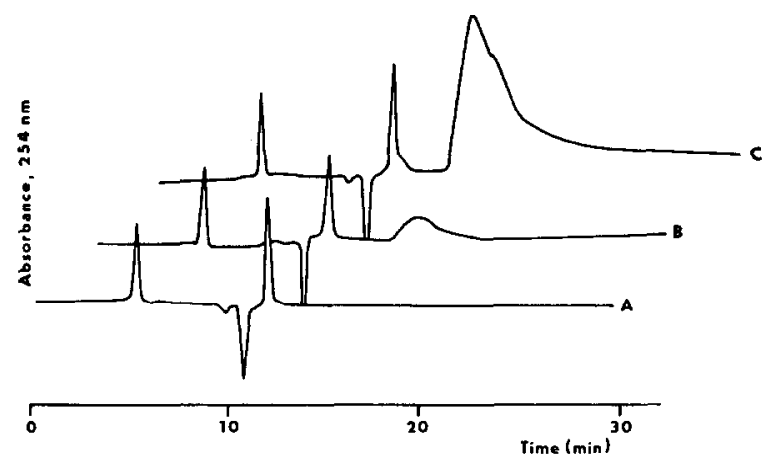

Fig. 1. Column chromatographic protiles obtained with different incubation mixtures involving AMP. (A) AMP alone; (B) AMP and ribose; (C) AMP, ribose and lysine (a.u.f.s. $=0.100$ ).

case of AMP at $12.2 \mathrm{~min}, \mathrm{CMP}$ at $19.8 \mathrm{~min}$ and GMP at $15.3 \mathrm{~min}$ ) represents free nucleoside, present as an impurity in the nucleotide sample.

As shown in Figs. 1B-3B, the new prominent peak occurred in all chromatograms, in which the reaction products of the nucleotide-ribose reaction were present. Its retention time was $27.0 \mathrm{~min}$ for CMP, $16.5 \mathrm{~min}$ for AMP and $17.0 \mathrm{~min}$ for GMP. The origin of the minor peak in Fig. $2 \mathrm{C}$ is unknown.

The peak of the nucleotide-sugar product was considerably increased if lysine was present in the incubation mixture (Figs. 1C-3C). It was established that the area of this peak represented $5-8 \%$ of the unreacted peak area of AMP (or other nucleotide involved). If lysine was replaced

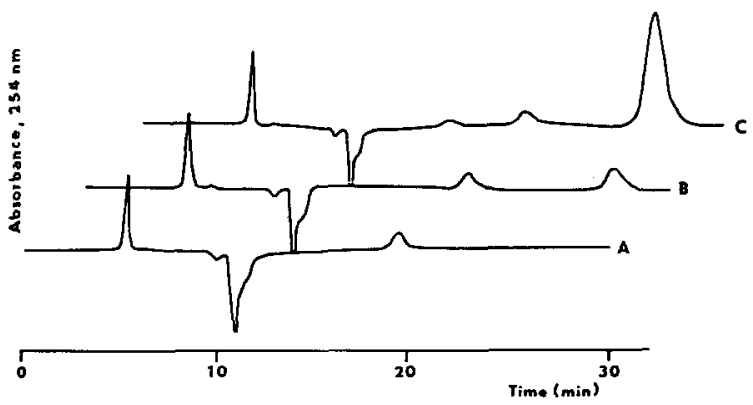

Fig. 2. Column chromatographic profiles obtained with different incubation mixtures involving CMP. (A) Nucleotide alone; (B) CMP and ribose; (C) CMP, ribose and lysine (a.u.f.s. $=0.100$ ).

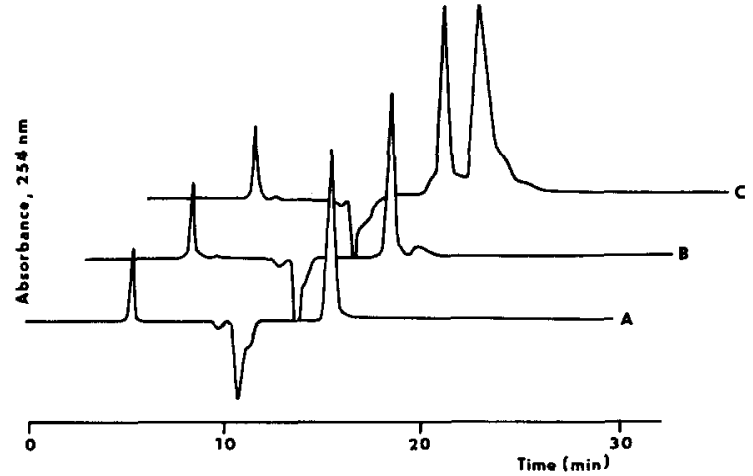

Fig. 3. Column chromatographic profiles obtained with different incubation mixture involving GMP. (A) Nucleotide alone; (B) GMP and ribose; (C) GMP, ribose and lysine (a.u.f.s. $=0.100$ ).

by another amino acid, e.g. arginine, $\beta$-alanine or $\mathrm{N}_{\varepsilon}$-acetyllysine, the resulting profiles were identical with those obtained with lysine (not shown). The retention time of the newly formed compound (adduct) depends solely on the nature of the nucleotide involved. Absorption spectra of all types of incubation mixture, e.g. AMP alone, AMP-ribose and AMP-ribose-lysine, showed a maximum at $260 \mathrm{~nm}$; if, however, the absorption spectra were measured by a diode-array detector during the chromatographic analysis, the peak in the reaction mixture where the amino acid (lysine) was present was shifted to $250 \mathrm{~nm}$ (Fig. 4). Obviously, the shift in absorption maxima can be seen only in the chromatographically separated

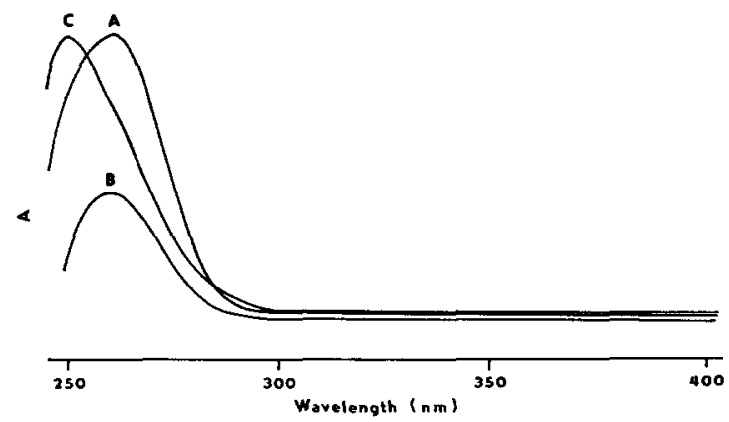

Fig. 4. Absorption spectra of particular peaks measured by diode-array detection. (A) Peak of AMP; (B) peak of AMPribose reaction product; (C) peak of AMP-ribose-lysine reaction product. The absorbance units (A) are different for different spectra. 
peak, as in the whole reaction mixture it is obscured by the excess of the unreacted nucleotide.

There are several ways in which an amino acid can be involved in the formation of the product. Either it can catalyse the reaction, e.g. by stimulating the formation of reactive intermediates in the nucleotide-sugar mixture, or it may alter the reaction pathway yielding a different product from that formed in the absence of an amino acid. In the latter case it must be assumed that the separation procedure used is not capable of separating the product(s) arising in the presence of an amino acid. On the other hand, as the reaction is dependent only on the nature of the nucleotide involved, it is feasible to assume that only a part, perhaps the $-\mathrm{NH}_{2}$ group of the amino acid, would be involved in the reaction. Of course, the best way of solving this dilemma would be to obtain a mass spectrum. This, however, is precluded by the fact that ethylene glycol must be used in the mobile phase to achieve elution (it is known that the Maillard reaction products tend to stick strongly to almost any kind of sorbent) which in the mass spectrum yields a high $-\mathrm{CH}_{2}$ signal resulting from impurities that are virtually impossible to remove. In addition the eluate is rich in dissolved silicic acid which represents another problem in mass spectral measurements.

Glucose and glyceraldehyde were also tested as glycating agents. The size of the peak obtained in the reaction between nucleotide (CMP), glucose and lysine was markedly lower than that obtained in the reaction mixture with ribose and lysine, and it was comparable in size with the peak occurring in the reaction of nucleotide and ribose alone (in the absence of any amino acid). This can be explained by the known differences in the reactivities of the two sugars. However, no similar peak in nucleotide-glyceraldehyde reaction sample was observed. The results were verified by TLC.

TLC separation (Fig. 5) shows the presence of single extra spot in the incubation mixtures with two different amino acids (lysine and alanine) as well as in the incubation mixture of AMP and ribose only. This strongly favours the hypothesis of a single product being formed in both the pres-

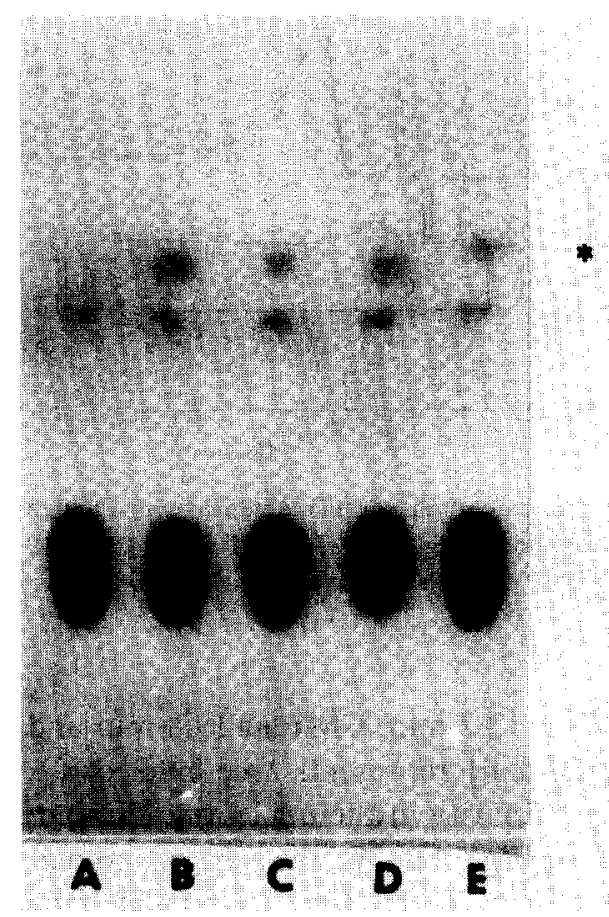

Fig. 5. Thin-layer chromatogram of reaction products formed from different reaction mixtures involving AMP. (A) AMP alone; (B) AMP, ribose and lysine; (C) AMP, ribose, and arginine; (D) AMP and ribose; (E) AMP, ribose and alanine. Asterisk indicates the reaction products formed.

ence and the absence of an amino acid in the incubation mixture. The small difference in the absorption maximum could be due to protonation of the product.

As formation of the nucleotide-sugar product in the presence of an amino acid is hindered by aminoguanidine, it appears feasible to assume the involvement of an Amadori intermediate. The inhibitory effect of aminoguanidine in protein-Maillard reaction can be explained in two ways: AG can react with the Amadori product and so inhibit its subsequent chemical transformation or it can trap dicarbonyl compounds formed during sugar autooxidation or Amadori product decomposition [20]. For instance, in the equimolar incubation mixture of an amino acid, reducing sugar and nucleotide, a part of the amino acid and reducing sugar may yield an Amadori product, which, being substituted in the presence of $\mathrm{AG}$, is prevented from bcing further in- 


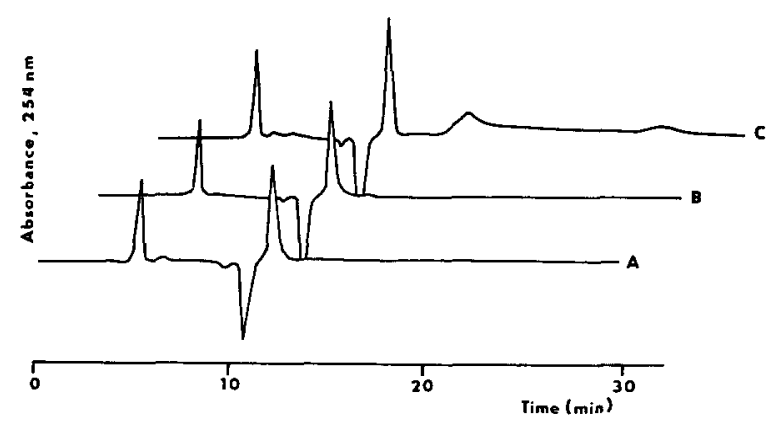

Fig. 6. Liquid column chromatography of the products arising from the reaction inhibited by diethylenetriamine pentaacetic acid (DETAPAC). (A) AMP with DETAPAC; (B) AMP with ribose and DETAPAC; (C) AMP with ribose, lysine and DFTA$\operatorname{PAC}$ (a.u.f.s. $=0.100$ ).

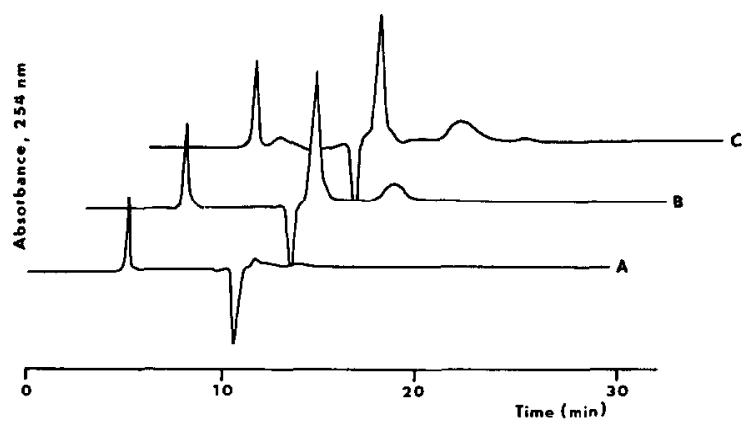

Fig. 7. Liquid column chromatography of the products arising from the reaction inhibited by aminoguanidine. (A) AMP with aminoguanidine; (B) AMP with ribose and aminoguanidine; (C) AMP with ribose, lysine and aminoguanidine (a.u.f.s. $=0.100$ ).

volved in the reaction with the nucleotide. It is therefore possible to propose that the formation of the nucleotide-sugar product involves reactive intermediates (deoxyosones), known to take part in the formation of advanced end glycosylation products [21].

On the other hand, the formation of the nucleotide-sugar product is blocked by DETAPAC as well. This means that formation of the sugarnucleotide reaction product should involve oxidative reactions catalysed by divalent transition metal cations (Figs. 6 and 7).

The nature of the Maillard reaction with nucleotides is still unknown. The products arising from the Maillard reaction involving nucleotides were thus far detected only on the basis of their spectral characteristics and their structure remains unclear. In this paper we present an attempt to separate the Maillard products from their unmodified counterparts.

\section{ACKNOWLEDGEMENTS}

We are grateful to Mr. M. Smrž for technical assistance. This work was supported by the Czechoslovak Academy of Sciences (Grant No. 71129).

\section{REFERENCES}

1 F. Saksela and P. S. Moorhead, Proc. Natl. Acad. Sci. U.S.A., 50 (1963) 390

2 G. B. Price, S. P. Modak and T. Makinodan, Science, 171 (1971) 917.

3 O. Yamamoto, I. Fuji, T. Yashida, A. B. Cox and J. T. Lett, J. Gerontol. Biol. Sci., 43 (1988) B132.

4 J. J. Bojanovic, A. D. Jevtovic, U. S. Pantic, S. Dugandzic and D. S. Javonovic, Gerontologia, 16 (1970) 304.

5 P. Karran and M. G. Ormerod, Biochim. Biophys. Acta, 299 (I973) 54

6 T. D. Petes, R. A. Farber, G. M. Tarrant and R. Holliday, Nature, 251 (1974) 434.

7 G. D. Bezdyshev and S. M. Zhelabovskaya, Exp. Gerontol., 7 (1972) 321.

8 R. Bucala, P. Model and A. Cerami, Proc. Natl. Acad. Sci. U.S.A., 81 (1984) 105.

9 R. Bucala, P. Model, M. Russel and A. Cerami, Proc. Natl. Acad. Sci. U.S.A., 82 (1985) 8439.

10 A. T. Lee and A. Cerami, Proc. Natl. Acad. Sci. U.S.A., 84 (1987) 8311.

11 A. T. Lee and A. Cerami, Mutat. Res., 179 (1987) 151.

12 T. K. Shires, J. Tresnak, M. Kaminsky, S. L. Herzog and B. Truc-Pham, FASEB J., 4 (1990) 3340.

13 S.-B. Kim, I.-S. Kim, D.-M. Yeum and Y.-M. Park, Mutat. Res., 254 (1991) 65.

14 T. Shibamoto, in G. Charalambons and G. Inglett (Editors), Instrumental Analysis of Food. Recent Progress, Academic Press, New York, 1983, p. 229.

15 H. Omura, N. Jahan, K. Shinohara and H. Murakami, in G. R. Waller and M. S. Feather (Editors), The Maillard Reaction in Food and Nutrition (ACS Symp. Ser., Vol. 215), American Chemical Society, Washington, DC, 1983, p. 537.

16 T. Yamaguchi and K. Nagakawa, Agric. Biol. Chem., 47 (1983) 2461.

17 C. K. Lim, in T. Hanai (Editor), Liquid Chromatography in Biomedical Analysis, Elsevier, Amsterdam, 1990, ch. 8, p. 191.

18 N.-I. Jang and P. R. Brown, in E. Heftmann (Editor), Chromatography, Elsevier, Amsterdam, 5th ed., 1992, ch. 17, p. B293.

19 V. D. Shatz and H. A. Kazoka, J. Chromatogr., 552 (1991) 23.

20 J. W. Baynes, Diabetes, 40 (1991) 405.

21 V. M. Monnier, J. Gerontol. Biol. Sci., 45 (1990) B105. 